\title{
Raman signatures of ferroic domain walls captured by principal component analysis
}

\author{
G F Nataf ${ }^{1,2,3,5}$,, N Barrett ${ }^{2}$, J Kreisel ${ }^{1,4}$ and M Guennou ${ }^{1}$ \\ 1 Materials Research and Technology Department, Luxembourg Institute of Science and Technology, 41 \\ Rue du Brill, L-4422 Belvaux, Luxembourg \\ 2 SPEC, CEA, CNRS, Université Paris-Saclay, CEA Saclay, 91191 Gif-sur-Yvette Cedex, France \\ ${ }^{3}$ Department of Materials Science, University of Cambridge, 27 Charles Babbage Road, Cambridge CB3 \\ 0FS, United Kingdom \\ ${ }^{4}$ Physics and Materials Science Research Unit, University of Luxembourg, 41 Rue du Brill, L-4422 \\ Belvaux, Luxembourg \\ E-mail: gn283@cam.ac.uk
}

Received 17 August 2017, revised 29 October 2017

Accepted for publication 1 November 2017

Published 18 December 2017

\begin{abstract}
Ferroic domain walls are currently investigated by several state-of-the art techniques in order to get a better understanding of their distinct, functional properties. Here, principal component analysis (PCA) of Raman maps is used to study ferroelectric domain walls (DWs) in $\mathrm{LiNbO}_{3}$ and ferroelastic DWs in $\mathrm{NdGaO}_{3}$. It is shown that PCA allows us to quickly and reliably identify small Raman peak variations at ferroelectric DWs and that the value of a peak shift can be deduced - accurately and without a priori-from a first order Taylor expansion of the spectra. The ability of PCA to separate the contribution of ferroelastic domains and DWs to Raman spectra is emphasized. More generally, our results provide a novel route for the statistical analysis of any property mapped across a DW.
\end{abstract}

Keywords: Raman, multivariate statistical analysis, domain wall

(Some figures may appear in colour only in the online journal)

Ever since it has been realized that domain walls (DWs) in ferroic materials can present novel structural properties that do not exist in the bulk domains, researchers have considered a new device paradigm where the DWs, rather than the domains, are the active element. This field has been coined 'Domain boundary engineering' $[1,2]$ or 'Domain wall nanoelectronics' [3]. The exploitation of the DWs nanometric size as well as their different functional properties presents a high potential for industrial innovation [2, 3]. Such functionalities include ferroelectric/polar DWs in ferroelastic $\mathrm{CaTiO}_{3}$ [4-6] or $\mathrm{SrTiO}_{3}$ [7], electric conductivity of domain walls in the insulating $\mathrm{BiFeO}_{3}$ [8-10] or $\mathrm{Pb}(\mathrm{Zr}, \mathrm{Ti}) \mathrm{O}_{3}$ [11-13], and photoinduced conductivity of DWs in lithium niobate $\left(\mathrm{LiNbO}_{3}, \mathrm{LN}\right)$ single crystals $[14,15]$. The example of local conductivity in the highly insulating and transparent $\mathrm{LN}$ is particularly appealing.

LN itself has been the subject of intensive studies for many years due to its ferroelectric, piezoelectric, pyroelectric,

\footnotetext{
${ }^{5}$ Author to whom correspondence should be addressed.
}

acoustic, electro-optical or photorefractive properties. It is suitable for applications in photorefractive devices, holographic memories, frequency doublers, etc [16]. At room temperature, it has a rhombohedral structure with space group $R 3 c$. Planes of oxygen atoms are arranged in a distorted hexagonal closepacked arrangement, with the interstices alternately filled by $\mathrm{Li}$ and $\mathrm{Nb}$ atoms. In the ferroelectric phase, $\mathrm{Li}$ and $\mathrm{Nb}$ atoms are slightly displaced from their position of high symmetry, defining the direction of the spontaneous polarization. The ferroelectric transition in $\mathrm{LN}$ is not ferroelastic_-as opposed to many classical ferroelectrics-which makes LN a model of the kind. Only two domain states are possible, both with polarization along the rhombohedral $c$-axis, and therefore only $180^{\circ}$-domain walls are observed. Interesting anomalies are observed in the vicinity of these DWs: unexpected optical contrast [17], strain on length scales of micrometers [18], anomalies in the Raman [19-25] and dielectric [26] spectra and photo-induced conduction $[14,15]$. These observations are usually discussed in term of variations in defect concentrations but the precise underlying mechanisms remain unclear 
and are debated in literature [19-21]. In the case of photoinduced conduction, an artefact effect due to the electrodes cannot be excluded.

Here, we present a study of DWs with Raman spectroscopy, which is a non-destructive, non-contact, and defectsensitive method for investigating ferroic materials. Generally speaking, the changes of the Raman spectra in the vicinity of DWs are small, with typical peak shifts below $1 \mathrm{~cm}^{-1}$. Thus, a large number of spectra and good statistics are usually needed for obtaining reliable results of the mostly very subtle changes. A further difficulty is that the typical ferroelectric or ferroelastic DW width is less than $10 \mathrm{~nm}$ [27], much less than the spatial resolution used in Raman spectroscopy. As a result, the Raman signal of interest is superimposed on a high background signal coming from the adjacent domains. The results are commonly analysed by peak fitting [21, 23-25] or by subtracting a spectrum at the domain wall from a spectrum far from it [20, 22] when Raman modes overlap, making fitting difficult. In this paper we demonstrate that a statistical analysis, namely principal component analysis (PCA) can overcome such difficulties and provides a rapid and robust way for the analysis of Raman data across DWs. Our work is inspired from literature reports on the use of PCA for Raman mapping of different chemical species [28, 29], to correct peak shifts smaller than the spectral resolution in Raman spectroscopy $[30,31]$, or even to detect a phase transition under pressure [32]. PCA has also been very useful in combination with a number of other experimental techniques: quantitative analysis of x-ray photoelectron spectroscopy imaging [33], scanning transmission electron microscopy image analysis [34], energy loss electron spectroscopy [35], etc. Here, we describe the interest of PCA for a quick and semi-quantitative analysis of DW signatures.

In the first part of this article we present simulations showing that PCA can be used to rapidly detect peak shifts, peak width and peak intensity variations in Raman spectra. In the second part, we apply PCA to the case of $180^{\circ}$-DWs in $\mathrm{LN}$ where contrast is only expected at the wall and compare the results with a standard fitting procedure. In the third part, we extend our discussion to variations of Raman modes at ferroelastic DWs in $\mathrm{NdGaO}_{3}$ where adjacent domains are also expected to contribute to contrast.

\section{Identification and localization of changes by PCA}

\subsection{Theory}

Raman measurements at domain walls usually consist of pointby-point mapping across a domain wall. The resulting maps typically contain hundreds of spectra. PCA is used to express the data in such a way as to highlight similarities and differences in a large set of spectral data. This can be used to identify specific spectral features otherwise hidden by the noise, or automatically identify characteristic signatures in a Raman map. These features are called principal components (PCs) [36]. Each spectrum $S$ obtained from the dataset, here a 3D matrix corresponding to a map of Raman spectra, can be described as a linear combination of PCs:

$$
S=S_{a}+\sum_{k} t_{k} \cdot \mathrm{PC}_{k}
$$

where $S_{a}$ is the mean spectrum calculated as the normalized sum of all the spectra and $t_{k}$ is a scalar, called the score, representing the weight of each component in the spectrum $S$. The number of PCs is equal to the number of spectra in the dataset.

These PCs are obtained from the singular value decomposition of the spectra in the mapping dataset $X$. Let us assume that $X$ is centred, i.e. column means have been subtracted and are now equal to zero. $X$ is expressed as the product of three matrices:

$$
X=U \cdot D \cdot W^{\mathrm{t}}
$$

where the columns of $U$ are the left-singular vectors of $X$, the diagonal entries of the diagonal matrix $D$ are the singular values of $X$ and the column of $W$ are the right-singular vectors of $X\left(W^{t}\right.$ is the conjugate transpose matrix of $\left.W\right)$. In the vocabulary of the PCA, the columns of $W$ are the PCs and the projections of the data on the PCs are the scores $t_{k}$ given by:

$$
X \cdot W=U \cdot D \cdot W^{\mathrm{t}} \cdot W=U \cdot D .
$$

The first $\mathrm{PC}\left(P C_{1}\right)$ is required to have the largest possible variance, in order to explain the most significant changes in the spectra. The second $\mathrm{PC}\left(\mathrm{PC}_{2}\right)$ is computed under the constraint of being orthogonal to the first $\mathrm{PC}$ and to have the largest possible variance. The other components are computed likewise [37].

The next stage of the analysis consists then in determining the number of components needed to describe the data: if too many PCs are retained, one might try to give a physical meaning to noise; if too few PCs are retained, one might miss essential information. A key parameter to determine the number of PCs is the proportion of total variance accounted for by each PC. This proportion $P$ is:

$$
P\left(\mathrm{PC}_{k}\right)=\frac{V\left(\mathrm{PC}_{k}\right)}{\sum V(S)}
$$

where $V(S)$ and $V\left(\mathrm{PC}_{k}\right)$ are the variances of each spectrum and each PC, respectively. The lower is $P$ the less information is contained in the PC. However, defining a threshold value for the number of PCs to be retained is arbitrary [28, 29]: the examples in figure 2 show that a PC which accounts for only $0.9 \%$ of the total variance can still have a physical meaning.

In Raman micro-spectroscopy, PCA has been commonly used for chemometric analysis. It allows to gain both spectral and spatial information when imaging various chemical species distributed over several micrometers [28, 29]. It has also been used to follow phase transitions [32]. In these cases, species or phases had well defined signatures and PCA produces components that can be directly interpreted as spectral signatures of chemical species. Conversely, in the case of $180^{\circ}$-DWs, the changes of the Raman spectra expected in the vicinity of the DW are small [19], leading to small alterations of the Raman spectra with respect to an average spectrum corresponding to the neighbouring domains. Thus the interpretation of the components is less straightforward. 


\subsection{Simulated DW Raman signatures}

To understand subtle changes and their signatures close to DWs, we perform simulations of Raman spectra with Python 2.7. We consider a series of 300 spectra containing each a single Lorentzian peak centred at a frequency of $750 \mathrm{~cm}^{-1}$, with a full width at half maximum (FWHM) of $20 \mathrm{~cm}^{-1}$ and an intensity $I$ of 100 . The discrete spectral resolution is of $1 \mathrm{~cm}^{-1}$. The spectrum number is associated to a spatial position $X$ (in $\mu \mathrm{m}$ ) along a line, i.e. one spectrum per micron. In order to determine the physical signification of these deviations, we classify them as peak shifts, FWHM changes and peak intensity variations. The objective of this part is to identify the characteristic shapes of the PCs associated to these spectrum perturbations.

In the first simulation, a DW is simulated by shifting the Raman peak in the central part of the line by $-0.5 \mathrm{~cm}^{-1}$ with respect to the two neighbouring 'domains', as shown in figure 1(a). PCA is applied to the series of simulated spectra: figure 1(d) shows the mean spectrum and the only PC obtained. This PC resembles the first derivative of the Lorentzian peak. Indeed, adding the derivative to the Lorentzian peak will increase the intensity on the left side of the peak maximum and decrease the intensity on the right side, shifting the Lorentzian to lower frequencies.

In the second simulation series, the FWHM of twenty Lorentzians is decreased by $2.5 \%$ before PCA is applied, as shown in figure 1(b). Figure 1(e) shows the PC obtained: it is symmetric with an increase of intensity at the centre. Adding the PC to the Lorentzian peak will decrease the intensity on its shoulders, reducing its FWHM.

We consider in the third simulation a series of spectra containing two Lorentzian peaks with the same intensity, centred at $400 \mathrm{~cm}^{-1}$ and $1200 \mathrm{~cm}^{-1}$. The intensity of the Lorentzian peak at higher frequency is progressively decreased by $0.5 \%$, decreasing the intensity ratio between the second and the first Lorentzian, as shown in figure 1(c). Figure 1(f) shows the mean spectrum and the PC obtained which resemble a Lorentzian peak with a negative intensity.

Figures 1(g)-(i) are the scores of the PCs for every spectrum. They reveal the spatial position of the twenty spectra affected by the changes. Qualitatively, the product of the PC and its score gives the 'trend' of the changes. As an example, the negative peak shift is characterized by an increase of the intensity of the PC on the left side of the Lorentzian maximum and a decrease on its right side-because the PC scores are positive.

\subsection{Influence of noise}

Because PCA is a statistical treatment, it is expected to be affected by the signal-to-noise ratio of the original dataset. We study the influence of noise in the following simulation. Twenty spectra are subjected to a shift of $+0.5 \mathrm{~cm}^{-1}$. A white noise with a normal distribution-centre at 0 and standard deviation $\sigma$-is added to every spectrum. We define the signal to noise ratio as $S N R=I / \sigma$, where $I$ denotes the intensity of the Lorentzian Raman peak.
As shown in figure 2(a), with $S N R=1000, P C_{1}$ still exhibits the expected characteristic shape and the score clearly shows the spatial position of the shifted spectra (figure 2(d)). With $S N R=100, P C_{1}$ is noisy and its score does not allow to define a precise value of the shift (figures 2(b) and (e)). With $S N R=10$, PCA is no longer able to detect the frequency shift (figure 2(c)) and the score reveals only the white noise (figure 2(f)). The absolute values of the scores increase when the $S N R$ decreases because of the higher variations in the intensity of the spectra.

The ability of PCA to detect subtle changes depends on their magnitude. Figure 3 shows how it is possible to detect a frequency shift, even with $\mathrm{SNR}=10$, by increasing the magnitude of the shift. When twenty Lorentzian are shifted by $3 \mathrm{~cm}^{-1}, P C_{1}$ resembles again the first derivative of the Lorentzian peak (figure 3(a)) but the score is noisy and does not allow a precise location of the shifted spectra (figure 3(d)). The contrast in the scores increases for frequency shift of $5 \mathrm{~cm}^{-1}$ (figure 3(e)) and $10 \mathrm{~cm}^{-1}$ (figure 3(f)). In other words, the SNR imposes a limit on the minimal peak shift that can be detected.

If the same white noise is added to every spectrum, PCA is able to distinguish it from the Lorentzian peak and to give an accurate value of the frequency shift (not shown). Any recurrent noise in a set of Raman spectra is therefore easily identified and filtered with PCA.

\subsection{Ability to identify several simultaneous changes}

Raman modes often undergo a peak shift and a FWHM change at the same time (for example when doping LN [19]). If the frequency and the FWHM of a Lorentzian peak are simultaneously modified, PCA still gives only one PC. In the example of figure 4 , a peak shift of $+0.5 \mathrm{~cm}^{-1}$ is applied combined with a decrease of the FWHM by $2.5 \%$. As shown in figure 4(a), this PC resembles a linear combination of the PCs obtained in figures 1(d) and (e). Figure 4(b) shows the score of the PC: the twenty modified Lorentzian peaks exhibit the same value. In other words, a simultaneous change of frequency and peak width is characterized by an asymmetric PC with maxima of opposite signs.

\section{Investigation of $180^{\circ}-\mathrm{DWs}$ : example of $\mathrm{LiNbO}_{3}$}

Ferroelectric DWs in lithium niobate with congruent $[19,20,22-24]$ or stoichiometric [20, 22, 25] compositions have been extensively studied by Raman spectroscopy [19, 20, 22-25]. The usual measurement consists of point-bypoint mapping across a DW. One reason for this interest is that $180^{\circ}$-domains by symmetry must have the same Raman spectrum. Any contrast observed near the DW can then be related to the DW itself. Peak shifts [19, 22, 25] and peak intensity variations [20, 21, 23-25] of the Raman modes have been observed but their relation to structure remains unclear. They are usually explained by defect concentration differences, or stress induced field variations. 

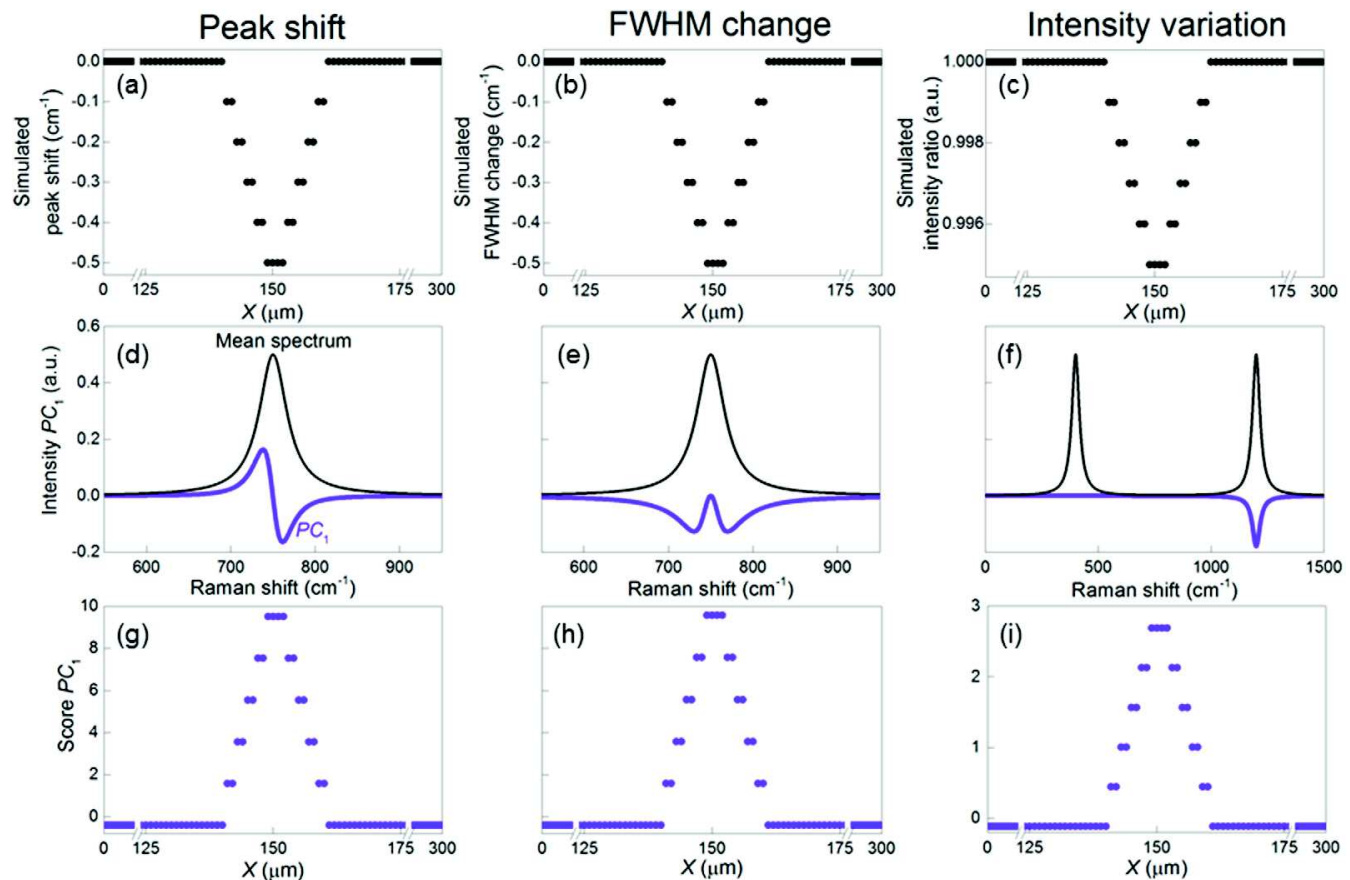

Figure 1. PCA results for simulated hypothetical spectra. Three hypothetical domain wall signatures are applied to 20 Lorentzian: (a) a shift of a single peak, (b) a change in the FWHM of a single peak and (c) an intensity variation between two peaks. The resulting signature of the principal component and mean spectrum are displayed in parts (d)-(f), while parts (g)-(i) present the corresponding scores.
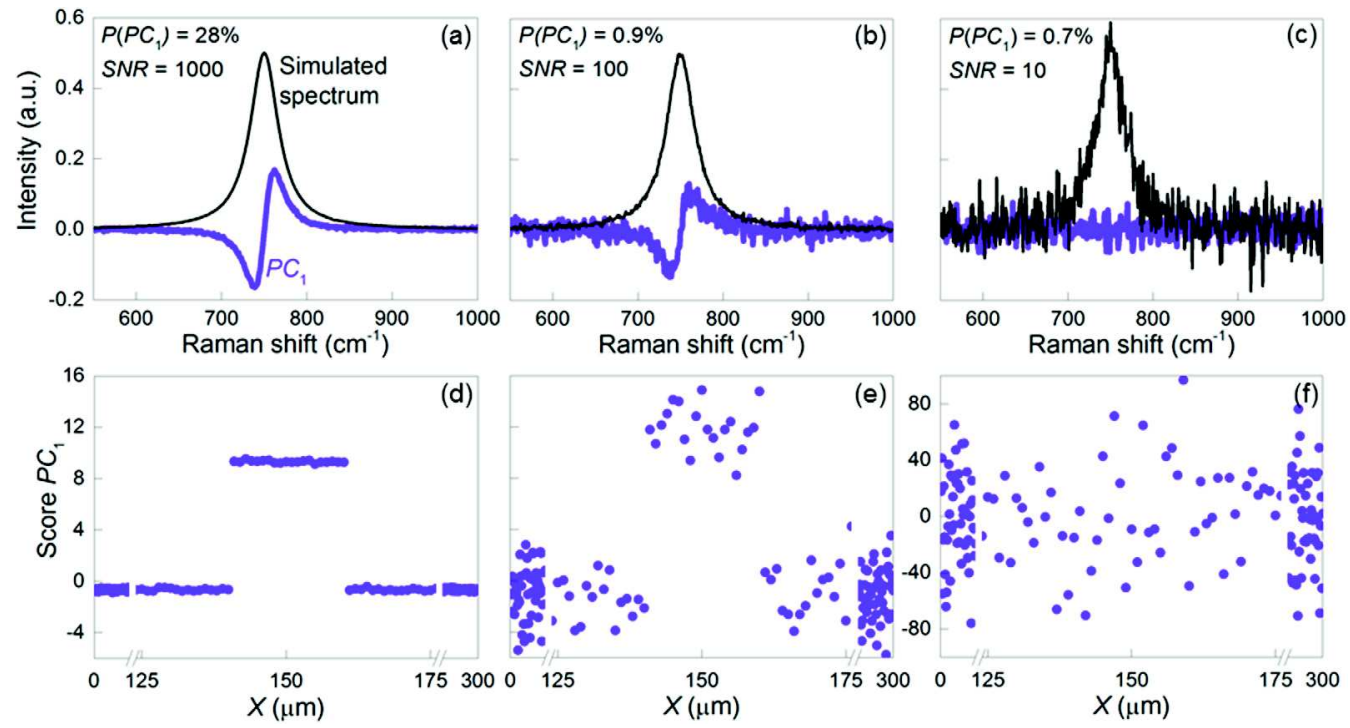

Figure 2. Influence of noise. $P C_{1}$ obtained when performing PCA on a peak shift of $+0.5 \mathrm{~cm}^{-1}$ with a SNR of (a) 1000 , (b) 100 and (c) 10 . (d)-(f) are the corresponding scores.

We investigated the $z$-face of a $5 \mathrm{~mol} \%$ magnesium doped lithium niobate single crystal. We performed a map of $2 \times 8 \mu \mathrm{m}^{2}$, in $0.1 \mu \mathrm{m}$ steps, as shown in the inset of figure 5(d). Each spectrum was acquired in $0.5 \mathrm{~s}$, with an exciting laser line at $442 \mathrm{~nm}$ and a spot size of $0.65 \mu \mathrm{m}$. After acquisition of the Raman map we used PCA to identify the main changes in the spectra near domain walls.

Figures 5(a)-(c) show three PCs and figures 5(d)-(f) the corresponding scores. In figures $5(\mathrm{a})-(\mathrm{c})$, the mean spectrum, corresponding to the sum of all the spectra in the data set, is also plotted for reference and is considered as the typical
Raman spectrum of LN. The number of PCs studied is limited to three because they account for $85 \%$ of the total variance. The fourth component-which account for only $0.04 \%$-and all the others contain only noise.

In figure 5(a), $P C_{1}$ is similar to the mean Raman spectrum. By direct comparison with the results of the simulation, this PC describes changes of intensity of the spectrum. These variations are usually referred to as 'noise' in Raman experiments: they are due to fluctuations of the laser intensity and drift of the sample holder during the measurement. Thus, the score of this PC is not at all correlated with the domain structure (figure 5(d)). 

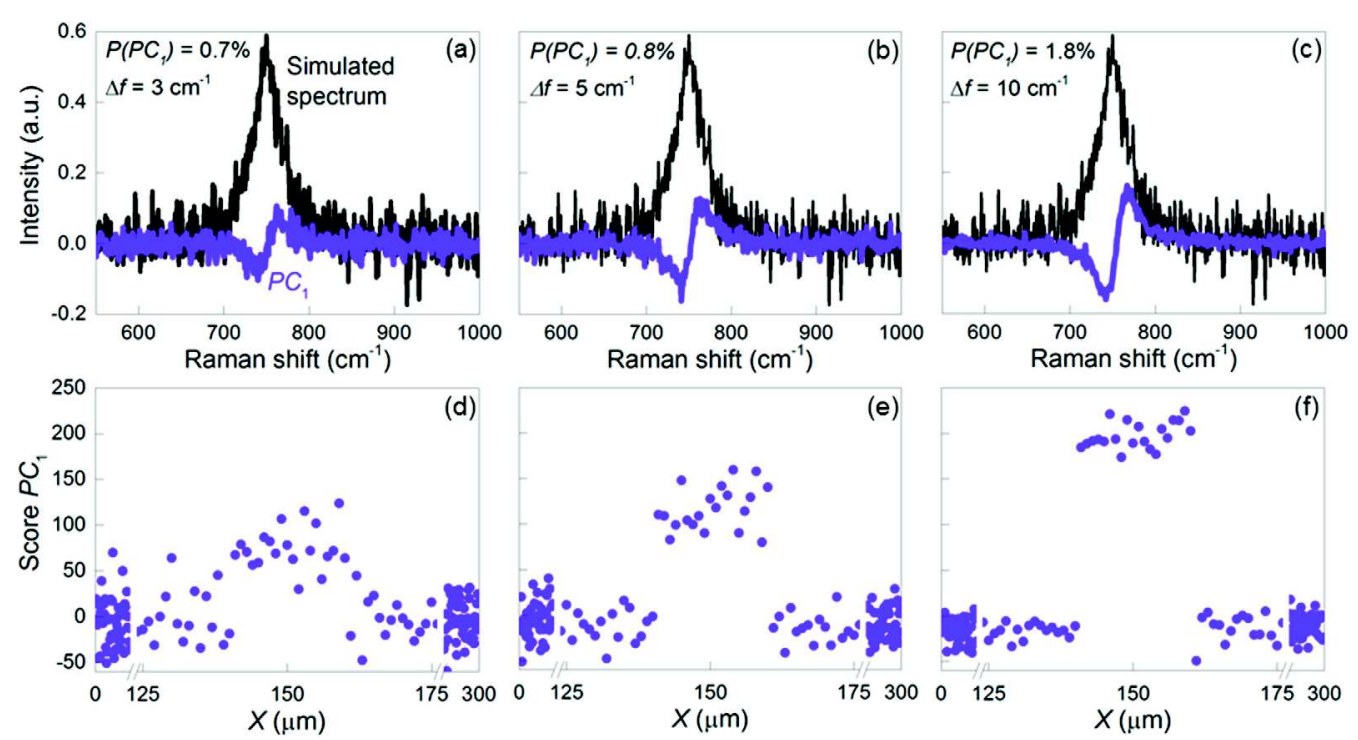

Figure 3. Influence of the amplitude of the frequency shift on noisy spectra. PC obtained when performing PCA on spectra with a SNR of 10 and a frequency shift of (a) $3 \mathrm{~cm}^{-1}$, (b) $5 \mathrm{~cm}^{-1}$ and (c) $10 \mathrm{~cm}^{-1}$. (d)-(f) are the corresponding scores for every spectrum.
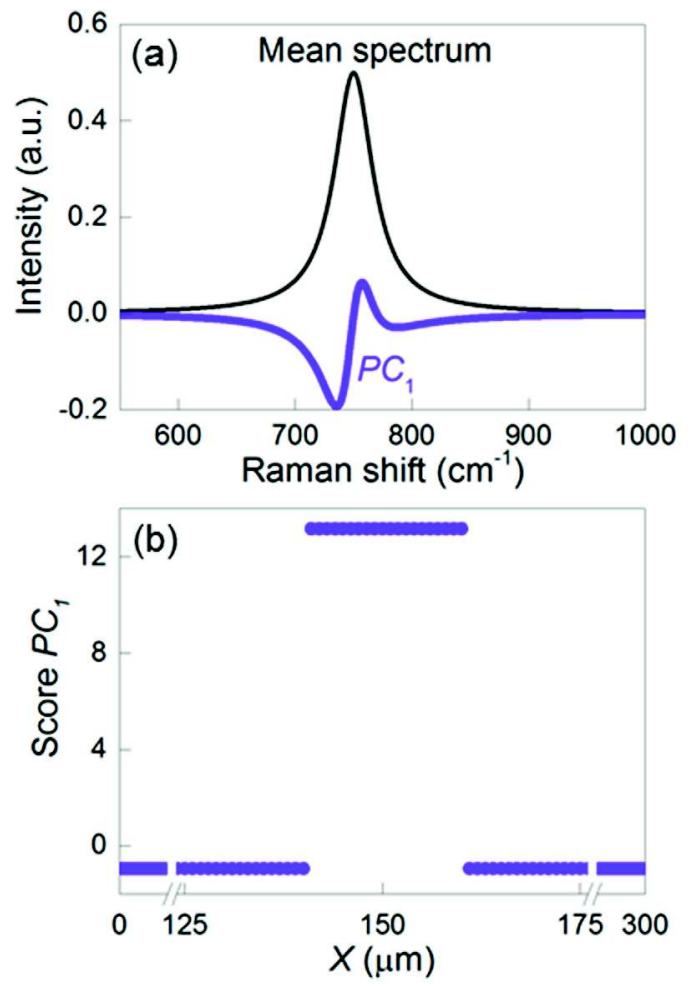

Figure 4. PCA on a simultaneous peak shift and FHWM variation. (a) Lorentzian peak unmodified and $P C_{1}$ (b) scores of $P C_{1}$.

Figures 5(b) and (c) present features centred at the same frequencies as the different Raman modes: they are similar to what was observed in the simulation of peak shifts or peak intensity variations. At $154 \mathrm{~cm}^{-1}, P C_{2}$ resembles the first derivative of a Lorentzian and by comparison with simulations, is interpreted as a shift of the $E$ (TO1) mode centred at $154 \mathrm{~cm}^{-1}$. By comparison with simulations, the large change of intensity of $P C_{2}$ near $615 \mathrm{~cm}^{-1}$ is a growing peak. $P C_{3}$ is dominated by two features: a shift of $E(\mathrm{TO} 1)$ at $154 \mathrm{~cm}^{-1}$ and a shift of $A_{1}(\mathrm{LO} 4)$ at $870 \mathrm{~cm}^{-1}$. Figures $5(\mathrm{~d})-(\mathrm{f})$ show the score of the first, second and third PCs, respectively. The score of $P C_{2}$ presents a high contrast in the position of the domain wall as determined by optical microscopy. Interestingly, the score of $\mathrm{PC}_{3}$ reveals a small contrast between domains which, by symmetry, is not expected. It is in fact due to the internal field of $\mathrm{LN}$ as explained below.

The interpretation of the PCA as performed on the full spectrum is that (i) the $E$ (TO1) peak position is slightly shifted between up- and down-polarized domains and shifts significantly at the domain wall (ii) the $A_{1}(\mathrm{LO} 4)$ peak position is different in domains. In order to study these two modes individually, we performed other PCAs whereby we restricted the frequency range to include only the peak of interest: between 40 and $200 \mathrm{~cm}^{-1}$ for $E$ (TO1), between 750 and $1000 \mathrm{~cm}^{-1}$ for $A_{1}(\mathrm{LO} 4)$. The first two PCs are shown in figure 6(a) for $E(\mathrm{TO} 1)$ and figure 6(b) for $A_{1}(\mathrm{LO} 4)$. They are similar to the features observed in figure 5 . For both modes, $P C_{1}$ resembles the Raman mode and $P C_{2}$ is similar to the derivative. The PCA shows that the sum of $P C_{1}$ and $P C_{2}$ describes well the $E(\mathrm{TO} 1)$ and $A_{1}(\mathrm{LO} 4)$ modes. Since $P C_{1}$ is similar to the mean signal, we can consider that $P C_{2}$ alone describes the frequency shift.

In order to compare the results of the PCA with the results of the standard fitting procedure, all spectra have been fitted with Voigt functions using the classical analysis approach. Figure 7(a) compares the normalized score of $P C_{2}$ with the normalized value of the $E$ (TO1) peak shift. The behaviour of $P C_{2}$ and the value of the peak shift are in good agreement. Figure 7(b) shows the same comparison for $A_{1}(\mathrm{LO} 4)$ mode. Again, a good agreement is observed.

By considering the true Raman spectrum as the sum of the mean spectrum and its derivative the spectra can be expanded in a Taylor series to first order:

$$
S(f)=b_{1} \cdot L(f)+b_{2} \cdot \frac{\mathrm{d} L}{\mathrm{~d} f}
$$



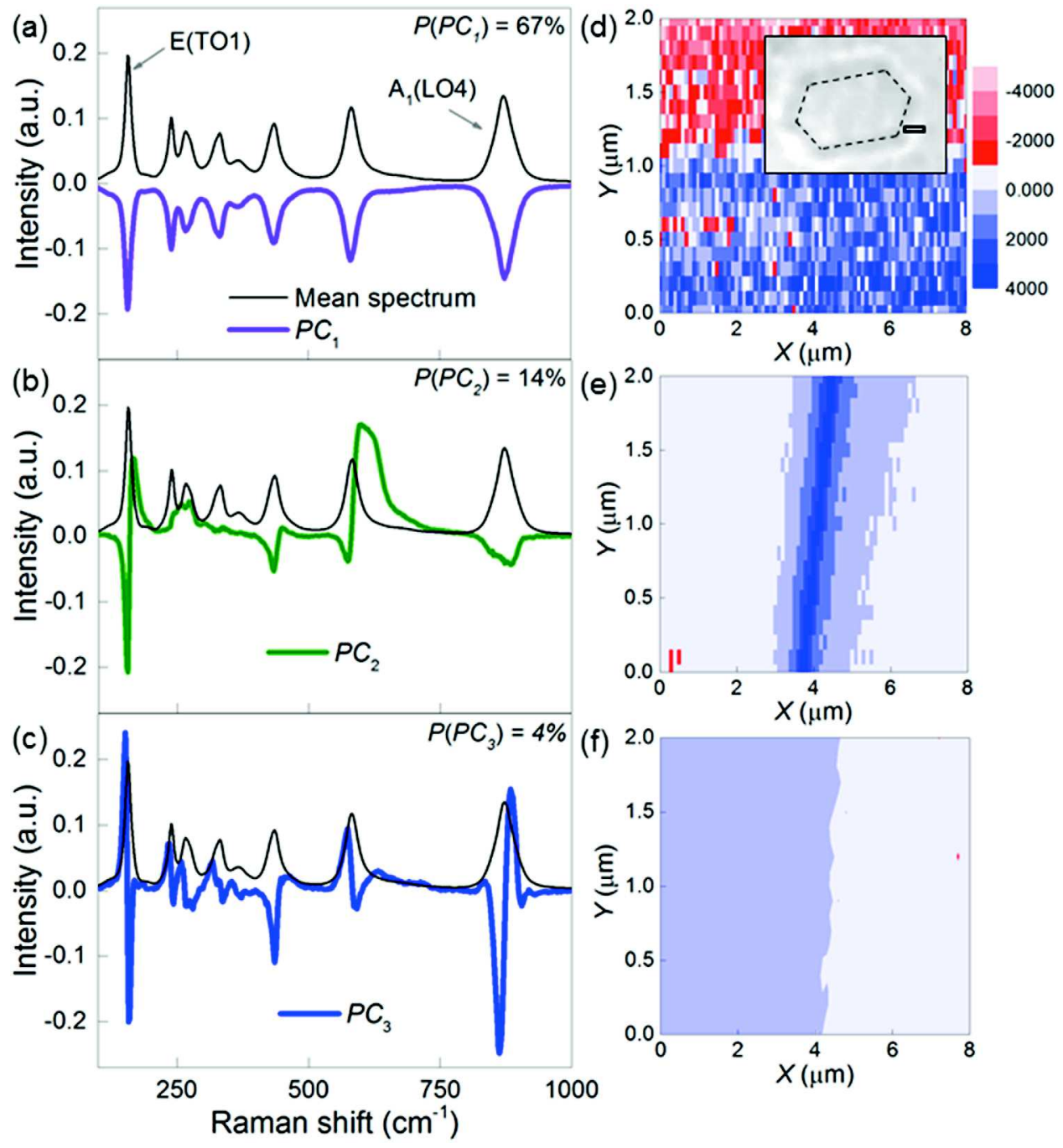

Figure 5. PCA Raman maps of ferroelectric domains and domain walls in $\mathrm{LiNbO}_{3}$. Mean spectrum and (a) first PC, (b) second PC, (c) third PC. (d)-(f) are the corresponding scores for every spectrum. The inset in (d) is an optical image of the domain with the investigated area indicated by the black rectangle and the DWs by dashed lines below them.

where $b_{1}$ and $b_{2}$ are the Taylor coefficients, $L$ is the lineshape function and $\frac{\mathrm{d} L}{\mathrm{~d} f}$ is the derivative of the spectrum with respect to the frequency $f$. Within the hypothesis of Lorentzian (or Voigt) lineshape:

$$
b_{1}=A \quad \text { and } \quad b_{2}=A \cdot \Delta f
$$

where $A$ is the peak maximum of the spectrum and $\Delta f$ is the peak shift. Thus, once a reference spectrum is chosen and its first derivative calculated, the Taylor coefficients can be estimated by linear regression of the reference spectrum and its derivative with respect to the complete series of spectra. The calculated regression coefficients are used to determine the peak shift $\Delta f$.

The full algorithm to determine the peak shift is the following:

(1) The mean spectrum is chosen as the reference spectrum and is normalized.

(2) Its first derivative is calculated numerically.

(3) Taylor coefficients are estimated by multiple linear regression. In matrix notation:

$$
B=\left(X^{\mathrm{t}} \cdot X\right)^{-1} \cdot X^{\mathrm{t}} \cdot S
$$

where the two columns of $X$ are the reference spectrum and its derivative, the columns of $S$ are the measured spectra pixel by pixel, the two columns of $B$ are the Taylor coefficients $\left(b_{1}, b_{2}\right)$.

(4) The frequency shifts are determined by:

$$
\Delta f=\frac{b_{2}}{b_{1}} .
$$

At the domain wall, a shift of $\sim 0.25 \mathrm{~cm}^{-1}$ is observed in $E$ (TO1) (figure 7(c)). It extends over $\sim 1.5 \mu \mathrm{m}$, which is about twice the theoretical spot size $(0.65 \mu \mathrm{m})$, i.e. more than one would expect from a typical $10 \mathrm{~nm}$ DW. While it is conceivable that modification of physical properties occurs on a larger scale than $10 \mathrm{~nm}$ (like strain fields near DWs in LN), there are also many experimental factors that can lead to a degradation of the instrumental response (slight defocusing, tiny misalignment of the sample, etc), and it is difficult to make 

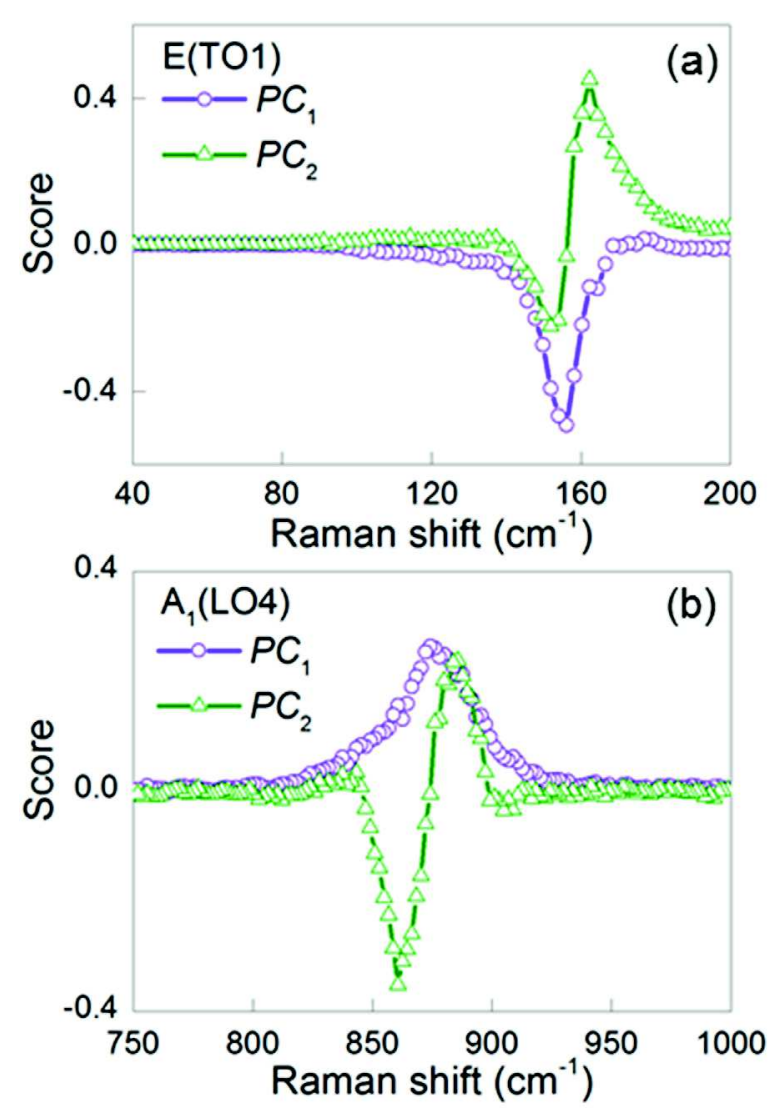

Figure 6. Results of the PCA analysis on individual modes. Scores of the first and second components for (a) $E$ (TO1) and (b) $A_{1}(\mathrm{LO} 4)$.

a conclusive statement about the true spatial extension of the effects. Furthermore, a shift of $\sim 0.1 \mathrm{~cm}^{-1}$ is observed between the domains. The behaviour of the calculated (PCA and Taylor expansion) and fitted (standard procedure) peak shifts are in good agreement: we obtain a slope of 1 with a Pearson's correlation coefficient of 0.99 when plotting one as a function of the other. Figure 7(d) shows the same comparison for $A_{1}(\mathrm{LO} 4)$ mode. A negative shift of $\sim 0.3 \mathrm{~cm}^{-1}$ is observed when going from the left to the right domain. The behaviour of the calculated and fitted peak shifts are again in good agreement (slope 1, Pearson's coefficient 0.99).

A previous systematic study of the influence of doping on the Raman signature at domain walls showed that (i) the frequency shift at the domain wall involves a combination of effects resulting from the defect structure and the electric (strain) field in the vicinity of the wall (ii) the Raman contrast that we observe between domains can be explained by the internal field induced by polar defect structures [19].

\section{Ferroelastic domain walls: example of $\mathrm{NdGaO}_{3}$}

Ferroelastic domains have by definition different crystallographic orientations that come with different orientations of the Raman tensor, which creates a contrast in Raman spectroscopy between two adjacent domains. This allows Raman mapping of ferroelastic domains, as is it customarily done [38]. Because of the large size of our laser spot $(\sim 0.65 \mu \mathrm{m})$ compared with the typical DW width $(<10 \mathrm{~nm})$, the Raman signal at the boundary between two ferroelastic domains contains a weighted average of the different Raman signal from both domains, possibly masking an extra, specific, signature of the DW. Identifying a specific signature of the DW is therefore much more challenging than in case of a pure ferroelectric DW.

In principle, the Raman contrast between the two domains cancels out for specific orientation and scattering geometries. This is a direct consequence of the strain compatibility condition obeyed by typical domains in bulk crystals. In practice though, it may be very difficult to identify and select this geometry. Besides, the cancellation would rely on a perfect realization of Raman selection rules, which are in reality only approximately fulfilled because of misorientations, optics, etc.

Here, we illustrate how PCA can be used to extract information from a Raman mapping across a ferroelastic domain wall. We investigate neodymium gallium oxide $\left(\mathrm{NdGaO}_{3}\right)$, which has an orthorhombic structure corresponding to the space group Pbnm, the most common structure among perovskites at room temperature [39], leading to 24 Raman modes among which 18 are well-resolved [40]. Its phonon dispersion curves and phonon eigenvectors have already been calculated [40]. The spontaneous strain coefficients, determined from the lattice parameters [41], are $e_{11}=8.2 \times 10^{-4}$ and $e_{12}=6.5 \times 10^{-3}$.

The Raman mapping of a $\mathrm{NdGaO}_{3}$ single crystal was carried out with a helium-neon laser at $633 \mathrm{~nm}$. Domains were observed with an optical microscope working with polarized light in order to select DWs almost orthogonal to the surface. We performed a map of $4 \times 16 \mu \mathrm{m}^{2}$, in $0.1 \mu \mathrm{m}$ steps, as shown in the inset of figure $8(\mathrm{c})$.

As shown in figure $8(\mathrm{c})$, the score of $P C_{1}$ is constant in the first domain, decreases on $\sim 3 \mu \mathrm{m}$ and reaches a new constant value in the second domain. The two scores have opposite sign. Thus, $P C_{1}$ describes the domain structure and the weighted average of the Raman signal from both domains. In figure 8(a), $P C_{1}$ is compared with two spectra extracted from the domains. $P C_{1}$ shows intensity variations: subtracting $P C_{1}$ from a spectrum of domain 1 gives a spectrum characteristic of domain 2 . $P_{2}$ must then describe an extra contribution observed in the vicinity of the wall, as evidenced by its score (figure $8(d)$ ). In figure $8(\mathrm{~b}), P C_{2}$ is compared with a spectrum from domain 1 . It exhibits mainly intensity variations of the Raman modes.

Thus, PCA allows Raman mapping of ferroelastic domains and DWs. Contrary to lithium niobate, where the contrast between domains was described by the PC with the smallest variance $\left(P C_{3}\right)$, the significant differences in intensities between the spectra of ferroelastic domains are described by $P C_{1}$, which accounts for $95 \%$ of the total variance. Ferroelastic DWs are oriented in such a way as to maintain strain compatibility between two adjacent domains, in order to minimize stress and elastic energy. Therefore, it is not surprising that we do not observe frequency shifts characteristic of strain variations at domain walls, contrary to DWs in lithium niobate where strain on length scales of micrometers have been reported [18]. It is not the purpose of this paper to give a detailed interpretation of the specific signature at the DW and how it relates to changes in structural and physical properties 

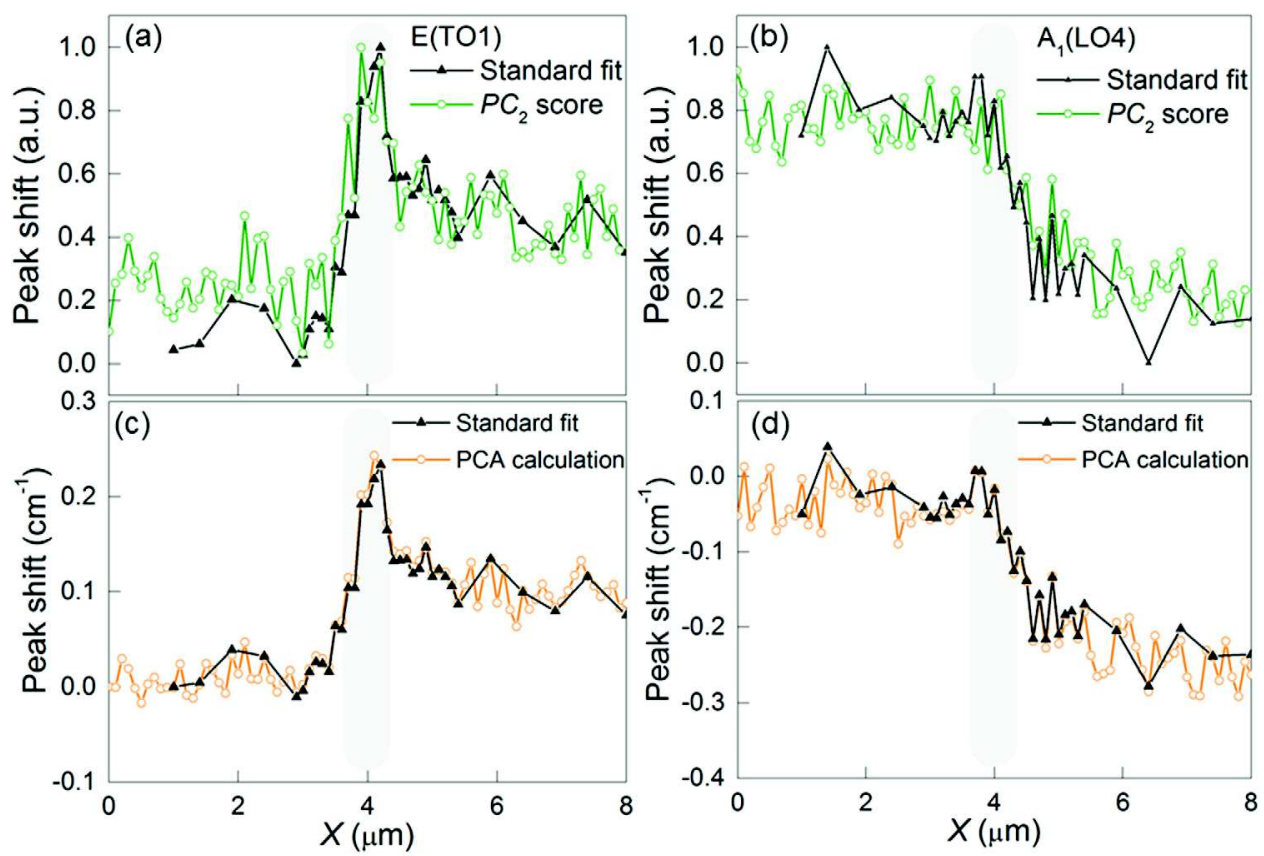

Figure 7. Peak shifts deduced from calculations and fitting procedures. Normalized components for (a) $E$ (TO1) and (b) $A_{1}(\mathrm{LO} 4)$. Absolute values for (c) $E$ (TO1) and (d) $A_{1}$ (LO4).
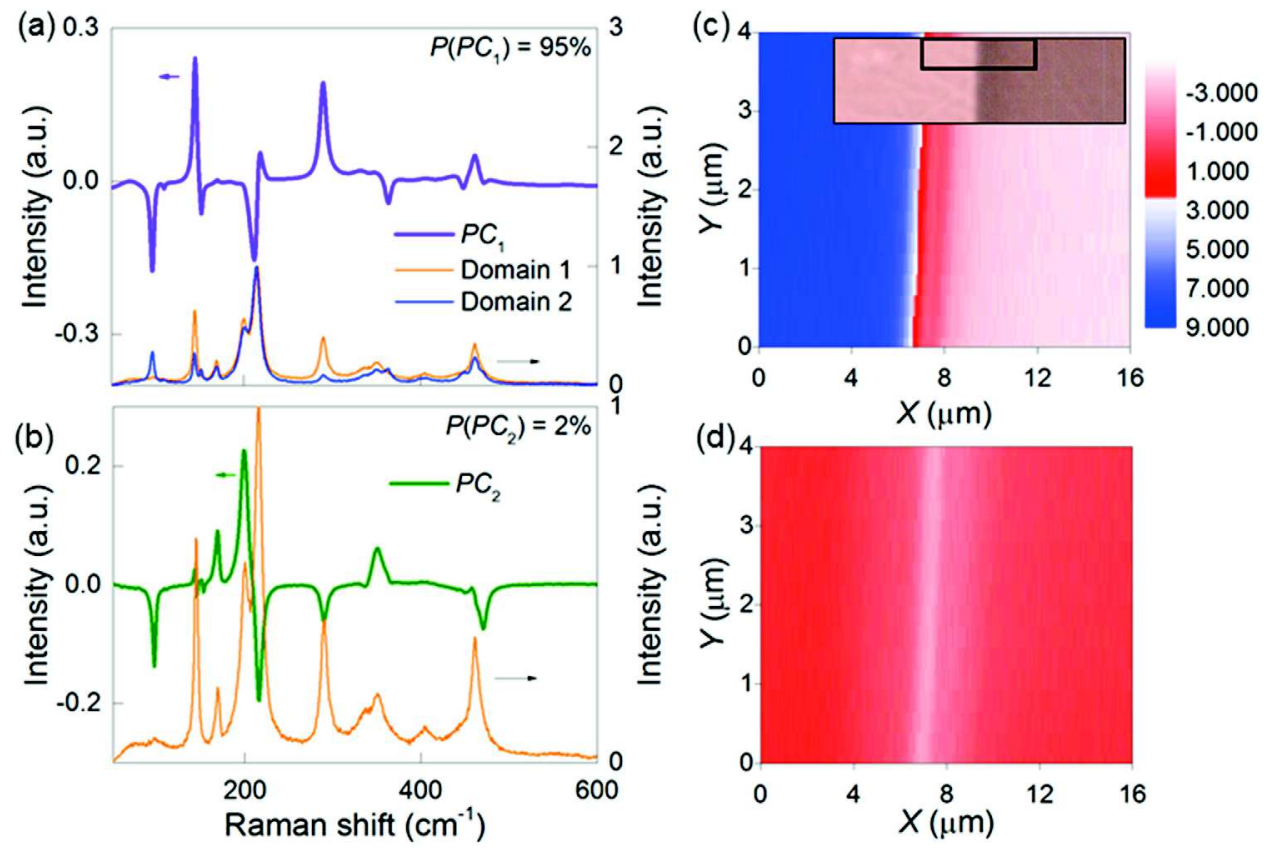

Figure 8. PCA Raman maps of ferroelastic domain and domain walls in $\mathrm{NdGaO}_{3}$. (a) Comparison of $P C_{1}$ and two spectra from domain 1 and domain 2. (b) Comparison of $P C_{2}$ with a spectrum from domain 1. (c) Scores of $P C_{1}$ for every spectrum. (d) Scores of $P C_{2}$ for every spectrum. The inset in (c) is an optical image of the domains with the investigated area indicated by the black rectangle.

(octahedral tilts, birefringence, etc); this is currently under investigation and requires more in-depth analyses.

\section{Conclusion}

In summary, our simulations show that PCA is a powerful tool to identify peak shifts, peak width and peak intensity variations in a 3D matrix corresponding to a map of Raman spectra. Raman measurements performed on $180^{\circ}$-DWs in lithium niobate evidence that PCA can be used to quickly identify and quantitively characterized small variations at DWs. PCA is also a promising tool to isolate the contribution of ferroelastic DWs.

Apart from DWs, Raman micro-spectroscopy and PCA could be used as complementary tools to map with a microscopic resolution the distribution of strain in materials, as required in silicon [42], graphene [43] or multilayer ceramic capacitors [44]. The use of PCA could also be extended to 3D matrix obtained through other experimental techniques, such as surface potential maps [45]. 


\section{Acknowledgments}

This work was supported by the Luxembourg National Research Fund (FNR) under project CO-FERMAT FNR/ P12/4853155/Kreisel.

\section{ORCID iDs}

G F Nataf (이 https://orcid.org/0000-0001-9215-4717

\section{References}

[1] Salje E K H and Zhang H 2009 Domain boundary engineering Phase Transit. 82 452-69

[2] Salje E K H 2010 Multiferroic domain boundaries as active memory devices: trajectories towards domain boundary engineering ChemPhysChem 11 940-50

[3] Catalan G, Seidel J, Ramesh R and Scott J F 2012 Domain wall nanoelectronics Rev. Mod. Phys. 84 119-56

[4] Van Aert S, Turner S, Delville R, Schryvers D, Van Tendeloo G and Salje E K H 2012 Direct observation of ferrielectricity at ferroelastic domain boundaries in $\mathrm{CaTiO}_{3}$ by electron microscopy Adv. Mater. 24 523-7

[5] Yokota H, Usami H, Haumont R, Hicher P, Kaneshiro J, Salje E K H and Uesu Y 2014 Direct evidence of polar nature of ferroelastic twin boundaries in $\mathrm{CaTiO}_{3}$ obtained by second harmonic generation microscope Phys. Rev. B 89144109

[6] Nataf G F et al (in press) Control of surface potential at polar domain walls in a nonpolar oxide Phys. Rev. Mater.

[7] Salje E K H, Aktas O, Carpenter M A, Laguta V V and Scott J F 2013 Domains within domains and walls within walls: evidence for polar domains in cryogenic $\mathrm{SrTiO}_{3}$ Phys. Rev. Lett. 111247603

[8] Seidel J, Fu D, Yang S-Y, Alarcón-Lladó E, Wu J, Ramesh R and Ager J W 2011 Efficient photovoltaic current generation at ferroelectric domain walls Phys. Rev. Lett. 107126805

[9] Seidel J et al 2009 Conduction at domain walls in oxide multiferroics Nat. Mater. 8 229-34

[10] Rojac T et al 2016 Domain-wall conduction in ferroelectric $\mathrm{BiFeO}_{3}$ controlled by accumulation of charged defects $\mathrm{Nat}$. Mater. 16 322-7

[11] Guyonnet J, Gaponenko I, Gariglio S and Paruch P 2011 Conduction at domain walls in insulating $\mathrm{Pb}\left(\mathrm{Zr}_{0.2} \mathrm{Ti}_{0.8}\right) \mathrm{O}_{3}$ thin films Adv. Mater. 23 5377-82

[12] Tselev A, Yu P, Cao Y, Dedon L R, Martin L W, Kalinin S V and Maksymovych P 2016 Microwave a.c. conductivity of domain walls in ferroelectric thin films Nat. Commun. 711630

[13] Jia C-L, Urban K W, Alexe M, Hesse D and Vrejoiu I 2011 Direct observation of continuous electric dipole rotation in flux-closure domains in ferroelectric $\mathrm{Pb}(\mathrm{Zr}, \mathrm{Ti}) \mathrm{O}_{3}$ Science 331 1420-3

[14] Schröder M, Haußmann A, Thiessen A, Soergel E, Woike T and Eng L M 2012 Conducting domain walls in lithium niobate single crystals Adv. Funct. Mater. 22 3936-44

[15] Schröder M, Chen X, Haußmann A, Thiessen A, Poppe J, Bonnell D A and Eng L M 2014 Nanoscale and macroscopic electrical ac transport along conductive domain walls in lithium niobate single crystals Mater. Res. Express 135012

[16] Arizmendi L 2004 Photonic applications of lithium niobate crystals Phys. Status Solidi $201253-83$
[17] Kim S and Gopalan V 2005 Optical index profile at an antiparallel ferroelectric domain wall in lithium niobate Mater. Sci. Eng. B 120 91-4

[18] Kim S, Gopalan V and Steiner B 2000 Direct x-ray synchrotron imaging of strains at $180^{\circ}$ domain walls in congruent $\mathrm{LiNbO}_{3}$ and $\mathrm{LiTaO}_{3}$ crystals Appl. Phys. Lett. 772051

[19] Nataf G F, Guennou M, Haußmann A, Barrett N and Kreisel J 2016 Evolution of defect signatures at ferroelectric domain walls in Mg-doped $\mathrm{LiNbO}_{3}$ Phys. Status Solidi 10 222-6

[20] Stone G and Dierolf V 2012 Influence of ferroelectric domain walls on the Raman scattering process in lithium tantalate and niobate Opt. Lett. 37 1032-4

[21] Zelenovskiy P S, Shur V Y, Bourson P, Fontana M D, Kuznetsov D K and Mingaliev E A 2010 Raman study of neutral and charged domain walls in lithium niobate Ferroelectrics 398 34-41

[22] Stone G, Lee D, Xu H, Phillpot S R and Dierolf V 2013 Local probing of the interaction between intrinsic defects and ferroelectric domain walls in lithium niobate Appl. Phys. Lett. 10242905

[23] Fontana M D, Hammoum R, Bourson P, Margueron S and Shur V Y 2008 Raman probe on PPLN microstructures Ferroelectrics 373 26-31

[24] Zelenovskiy P S, Fontana M D, Shur V Y, Bourson P and Kuznetsov D K 2010 Raman visualization of micro- and nanoscale domain structures in lithium niobate Appl. Phys. A $99741-4$

[25] Taniguchi H, Fujii Y and Itoh M 2013 Confocal micro-Raman imaging on $180^{\circ}$-domain structure in periodically poled stoichiometric $\mathrm{LiNbO}_{3}$ J. Ceram. Soc. Japan 121 579-82

[26] Nataf G F, Aktas O, Granzow T and Salje E K H 2016 Influence of defects and domain walls on dielectric and mechanical resonances in $\mathrm{LiNbO}_{3}$ J. Phys.: Condens. Matter 2815901

[27] Gonnissen J, Batuk D, Nataf G F, Jones L, Abakumov A M, Van Aert S, Schryvers D and Salje E K H 2016 Direct observation of ferroelectric domain walls in $\mathrm{LiNbO}_{3}$ : wall-meanders, kinks, and local electric charges $A d v$. Funct. Mater. 26 7599-604

[28] Vajna B, Patyi G, Nagy Z, Bódis A, Farkas A and Marosi G 2011 Comparison of chemometric methods in the analysis of pharmaceuticals with hyperspectral Raman imaging J. Raman Spectrosc. 42 1977-86

[29] Idarraga I, Mermoux M, Duriez C, Crisci A and Mardon J P 2012 Potentialities of Raman imaging for the analysis of oxide scales formed on zircaloy- 4 and $\mathrm{M} 5{ }^{\circledR}$ in air at high temperature Oxid. Met. 79 289-302

[30] Witjes H, van den Brink M, Melssen W J and Buydens L M 2000 Automatic correction of peak shifts in Raman spectra before PLS regression Chemom. Intell. Lab. Syst. 52 105-16

[31] Witjes H, Pepers M, Melssen W J and Buydens L M C 2001 Modelling phase shifts, peak shifts and peak width variations in spectral data sets: its value in multivariate data analysis Anal. Chim. Acta 432 113-24

[32] Sato-Berrú R Y, Mejía-Uriarte E V, Frausto-Reyes C, Villagrán-Muniz M, Murrieta H and Saniger J M 2007 Application of principal component analysis and Raman spectroscopy in the analysis of polycrystalline $\mathrm{BaTiO}_{3}$ at high pressure Spectrochim. Acta A 66 557-60

[33] Béchu S, Richard-Plouet M, Fernandez V, Walton J and Fairley N 2016 Developments in numerical treatments for large data sets of XPS images Surf. Interface Anal. 48 301-9

[34] Borisevich A et al 2010 Mapping octahedral tilts and polarization across a domain wall in $\mathrm{BiFeO}_{3}$ from Z-contrast scanning transmission electron microscopy image atomic column shape analysis ACS Nano 4 6071-9 
[35] Bosman M, Watanabe M, Alexander D T L and Keast V J 2006 Mapping chemical and bonding information using multivariate analysis of electron energy-loss spectrum images Ultramicroscopy 106 1024-32

[36] Abdi H and Williams L J 2010 Principal component analysis Wiley Interdiscip. Rev. Comput. Stat. 2 433-59

[37] Cangelosi R and Goriely A 2007 Component retention in principal component analysis with application to cDNA microarray data Biol. Direct 22

[38] Nakamura M, Orihara H, Ishibashi Y and Hara K 1990 Observation of ferroelastic domains in $\mathrm{LaNbO}_{4}$ by microRaman spectroscopy J. Phys. Soc. Japan 59 4472-5

[39] Lufaso M W and Woodward P M 2001 Prediction of the crystal structures of perovskites using the software program SPuDS Acta Crystallogr. B 57 725-38

[40] Kamishima O, Koyama H, Takahashi R, Abe Y, Sato T and Hattori T 2002 Raman study on symmetry analysis in $\mathrm{NdGaO}_{3}$ J. Phys.: Condens. Matter 14 3905-19
[41] Marti W, Rivera J-P, Kubel F, Scheel H J and Schmid H 1995 Optical studies of ferroelastic domain structures of $\mathrm{LaGaO}_{3}$ and $\mathrm{NdGaO}_{3}$ Ferroelectrics 172 79-84

[42] De Wolf I 1996 Micro-Raman spectroscopy to study local mechanical stress in silicon integrated circuits Semicond. Sci. Technol. 11 139-54

[43] Yu T, Ni Z, Du C, You Y, Wang Y and Shen Z 2008 Raman mapping investigation of graphene on transparent flexible substrate: the strain effect J. Phys. Chem. C 112 12602-5

[44] Nishida K, Kishi H, Funakubo H, Takeuchi H, Katoda T and Yamamoto T 2007 Evaluation of residual strain and oxygen vacancy in multilayer ceramic capacitor using laser raman spectroscopy Japan J. Appl. Phys. 46 7005-7

[45] Nataf G F, Grysan P, Guennou M, Kreisel J, Martinotti D, Rountree C L, Mathieu C and Barrett N 2016 Low energy electron imaging of domains and domain walls in magnesium-doped lithium niobate Sci. Rep. 633098 